BMJ Open Sport \& Exercise Medicine

\section{Conservative treatment of anterior chronic exertional compartment syndrome in the military, with a mid- term follow-up}

To cite: Zimmermann WO, Hutchinson MR, Van den Berg R, et al. Conservative treatment of anterior chronic exertional compartment syndrome in the military, with a midterm follow-up. BMJ Open Sport \& Exercise Medicine 2019;5:e000532. doi:10.1136/ bmjsem-2019-000532

- Additional material is published online only. To view please visit the journal online (http://dx.doi.org/10.1136/ bmjsem-2019-000532).

Accepted 21 February 2019

Check for updates

(C) Author(s) (or their employer(s)) 2019. Re-use permitted under CC BY-NC. No commercial re-use. See rights and permissions. Published by BMJ

For numbered affiliations see end of article.

Correspondence to Dr Wes 0 Zimmermann; wesselzimmermann@hotmail. com

\section{ABSTRACT}

Objectives To assess the outcome of conservative treatment for chronic exertional compartment syndrome (CECS) as it relates to the reduction in surgical fasciotomy and return to active duty in a military population. Methods Historic cohort. From 2015 to 2018, 75 surgically eligible patients with pressure-positive anterior CECS (Group 1), or with positive pressures and associated medial tibial stress syndrome (Group 2), underwent a conservative treatment programme emphasising gait retraining of running and marching. Treatment success was defined as return to duty, without surgery. Fifty patients from 2015 to 2017 were surveyed to assess midterm outcomes.

Results The average duration of conservative treatment was $144.9( \pm 59.6)$ days. Initially, $65 \%(49 / 75)$ were able to return to duty; $28 \%(21 / 75)$ were referred for surgery and $7 \%(5 / 75)$ left the armed forces. There was no difference in outcomes between Group 1 and Group 2. Survey response rate, on average after 742 days (SD 267, range 381-1256), was $84 \%$ (42/50); $57 \%$ (24/42) had continued duty, without surgery; of them, $43 \%$ were at the same military specialty, $57 \%$ in a physically less demanding job. Conclusion A conservative treatment programme for anterior CECS was able to return $65 \%$ of patients to active duty, without surgery. At 2 years, the success rate decreased slightly, but remained positive at $57 \%$. In this high-risk group, initiating a conservative treatment protocol with an emphasis on gait retraining can significantly reduce the need for surgical fasciotomy. For those that fail conservative treatment, surgical release may still be indicated.

\section{INTRODUCTION}

Chronic exertional compartment syndrome (CECS) is one of the causes of exercise-related leg pain in athletes and service members. A clinical description of CECS is repetitive pain and pathologically elevated pressure in a muscular compartment during physical exercise, which returns to normal with cessation of exercise. ${ }^{1}$ CECS can present in any muscular compartment of the human body, but is most prevalent in the anterior compartment of the

\section{What are the new findings?}

This study reports promising results of a special conservative treatment programme for anterior chronic exertional compartment syndrome in the military.

- This study presents the largest case series published until now $(n=75)$.

- This study reports the longest follow-up until now ( $>2$ years)

How might this impact on clinical practice in the future?

In the military, treatment for anterior chronic exertional compartment syndrome may be initiated with a special conservative approach before fasciotomy.

- The conservative treatment programme should at least contain gait retraining of running and marching, in shoes and boots.

- The number of surgical procedures can be reduced significantly; however, surgery is still indicated when conservative treatment is not effective.

leg (anterior CECS). ${ }^{2}$ The incidence of CECS in the USA armed forces is estimated at 0.49 cases per 1000 person years (4100 cases diagnosed in 5 years). ${ }^{3}$

Several authors have claimed that non-operative treatment of CECS rarely leads to complete resolution of symptoms or return to previous levels of athletic or military activity; thus, surgery (fasciotomy) has been strongly recommended as first-line treatment and been termed the gold standard of treatment. ${ }^{45}$ Unfortunately, it has also been recognised in a number of studies that the results of fasciotomy and return to former activity level are less favourable in the military population. ${ }^{1356}$ In the Central Military Hospital, Utrecht, The Netherlands, every patient with anterior CECS and a positive pressure test received fasciotomy without delay for many years. ${ }^{7}$ Unfortunately, the 
occupational prognosis after surgery proved to be poor: A 2-year follow-up of 44 patients with anterior CECS after surgery showed that only 15 patients $(34 \%)$ had returned to their original military jobs, 28 patients $(64 \%)$ had left the military and 25 patients $(57 \%)$ still had symptoms of exercise-related leg pain. ${ }^{8}$ Therefore, the Central Military Hospital changed its policy regarding the protocol of surgical indication. From 2011 onward, patients with clinically proven anterior CECS and a positive pressure test, that is, eligible for surgery, were sent first to a comprehensive conservative treatment programme, either in the Military Rehabilitation Center (Doorn, The Netherlands) or the department of Military Sports Medicine (Utrecht, The Netherlands). Referral was not truly random, and more complicated cases were sent to the Military Rehabilitation Center. This centre offered an inpatient conservative treatment programme containing physical therapy, physical fitness, mental coaching and podiatry. The results were described in two studies: $56 \%$ of patients were able to return to base in order to resume military duties without surgery, and the others returned to the surgeon. ${ }^{910}$ The results of the conservative treatment programme of the department of Military Sports Medicine were unknown until now. An evaluation is important to determine whether delaying surgery is justified.

The purpose of this study was to determine the initial and mid-term follow-up results of the comprehensive conservative treatment programme in service members with clinically proven anterior CECS and a positive pressure test offered at the department of Military Sports Medicine.

\section{MATERIAL AND METHODS}

This study is a historic cohort, involving patients seen by the first author (WZ) in the Central Military Hospital and in the department for Military Sports Medicine, with a follow-up survey, minimally 1 year after completion of a special conservative treatment programme.

\section{Organisation of care}

The Royal Netherlands Armed Forces have a diagnostic and treatment protocol for exercise-related leg pain coordinating physicians and physical therapists working in outlying primary care clinics with specialists in the Central Military Hospital. This protocol describes that service members with exercise-related leg pain be referred to the Central Military Hospital if conservative therapy in primary care has not been successful within 6 months. ${ }^{8}$ Since 2013 , this hospital has offered a specialty clinic for service members with exercise-related leg pain. A multidisciplinary team of surgery, sports medicine and physiatry evaluates patients in a one-stop shop setting. Diagnostic imaging is ordered if stress fractures, malign or vascular disorders have to be excluded. The latter is in less than $5 \%$ of cases. After medical clearance, a sports medicine physician supervises the patient in a standardised running test on a treadmill to pain tolerance and performs an intracompartmental pressure measurement of compartments suspected for CECS. The standardised running test, the minute-by-minute pain scoring system, the exact execution of the pressure measurement with a Stryker needle manometer and the diagnostic flowchart for exercise-related leg pain were described previously in detail and are available here as online supplementary material $1-3 .^{11}$ All patient information is stored in an electronic patient record. Based on the evaluations, patients may be referred to conservative treatment in the Military Rehabilitation Center (inpatient) or the Department of Military Sports (outpatient) or to fasciotomy in house. The criteria for surgery for anterior CECS are pain with exertion in the anterior compartment of the leg and pressure $\geq 35 \mathrm{~mm} \mathrm{Hg}$ in the first minute postexercise and were established locally. ${ }^{7}$

\section{Inclusion}

Medical records at the department of Military Sports Medicine were searched for all patients diagnosed with anterior CECS (Group 1) and anterior CECS+medial tibial stress syndrome (MTSS) (Group 2) sent from the Central Military Hospital in the years 2015-2018. The following baseline information was obtained: age (years), sex (male/female), height $(\mathrm{m})$, weight $(\mathrm{kg})$, body mass index, diagnosis (anterior CECS or anterior CECS+MTSS), intracompartmental pressure measurement values, duration of symptoms (months), repeat episode (yes/no), symptoms in first year of service (yes/ no), the Single Assessment Numerical Evaluation (SANE) score at intake. This SANE score is a single question instrument evaluating patients' subjective injury status with the following question: 'how would you rate your lower leg today as a percentage of normal, on a $0-100$ scale, with 100 being normal'. The SANE score was developed and validated in a military healthcare setting. ${ }^{12}$

\section{Comprehensive conservative outpatient treatment programme} At the department of Military Sports Medicine, each patient received a comprehensive conservative outpatient treatment programme with a selection of interventions described in the literature: ${ }^{13}{ }^{14}$ stretching and/or strengthening of lower extremity musculature, massage of hypertonic musculature, dry needling of trigger points, extracorporeal shockwave therapy to the medial tibial border, supplementation of vitamin $\mathrm{D}$, evaluation of running shoes, evaluation of shoe inlays, maintaining fitness with a low impact training programme, gait retraining of running and marching and a progressive running schedule. For each of the aforementioned interventions, local protocol describes criteria for application (table 1). If patients received extracorporeal shockwave therapy for MTSS (once per week, four sessions), gait retraining was postponed for the duration of this treatment. Once free of pain in rest, every service member received a 6-week progressive running programme building up to a 15 min uninterrupted run. Some service members with a physically demanding specialty, received 
Table 1 Standard care for exercise-related leg pain: criteria for application (version 2018)

\begin{tabular}{ll}
\hline Intervention & Criterion \\
\hline Stretching & $\begin{array}{l}\text { Gastrocnemius tightness=minimal angle } \\
\text { compared with a vertical line: } 70^{\circ} \text { or more. } \\
\text { Soleus tightness=maximal distance of the } \\
\text { big toe } 5 \mathrm{~cm} \text { from the wall or less. } \\
\text { See online supplementary material 4, with } \\
\text { two illustrations. }\end{array}$ \\
Strengthening & $\begin{array}{l}\text { Calve strength insufficient: not able to } \\
\text { perform } 30 \text { consecutive calve raises on } \\
\text { one leg. }\end{array}$ \\
$\begin{array}{l}\text { Massage } \\
\text { hypertonic m. } \\
\text { plantaris }\end{array}$ & $\begin{array}{l}\text { m. plantaris palpation painful (patient in } \\
\text { prone position). }\end{array}$ \\
$\begin{array}{l}\text { Dry needling of } \\
\text { trigger points }\end{array}$ & $\begin{array}{l}\text { Medial and lateral gastrocnemius: if } \\
\text { patient identifies the calve as a pain } \\
\text { location. }\end{array}$ \\
$\begin{array}{l}\text { Compression } \\
\text { stockings/ } \\
\text { sleeves }\end{array}$ & $\begin{array}{l}\text { Not given to patients with proven anterior } \\
\text { CECS (ICPM } \geq 35 \text { mm Hg). }\end{array}$
\end{tabular}

ESWT

For MTSS only: once a week, 4 sessions; each session 2000 radial shocks, frequency eight per second and intensity 2.5 bar, on the medial tibial border.

\begin{tabular}{|c|c|}
\hline $\begin{array}{l}\text { Vitamin D } \\
\text { supplementation }\end{array}$ & $\begin{array}{l}\text { If MTSS is present: criterion: }<50 \mathrm{nmol} / \mathrm{L} \\
\text { means insufficient, supplementation } \\
\text { required; optimal } 75 \mathrm{nmol} / \mathrm{L} \text {. }\end{array}$ \\
\hline $\begin{array}{l}\text { New running } \\
\text { shoes }\end{array}$ & $\begin{array}{l}\text { Every year or } 500 \text { miles }(800 \mathrm{~km}) \text {. } \\
\text { If patient describes a relation between } \\
\text { symptoms and shoes. } \\
\text { Minimalist shoes are discouraged. }\end{array}$ \\
\hline
\end{tabular}

Customised If navicular drop is positive $(>0.5 \mathrm{~cm})$ and antipronation if overpronation is established with slow inlays motion video analysis of barefoot running.

Maintaining fitness with low impact training

Resume three moments of low impact exercise per week. Keep leg pain scores $\leq 3$ (on a Numeric Pain Rating Scale 0-10).

Gait retraining while running in sports shoes and boots

\section{Four cues for running:}

1. Change to a ball-of-foot strike (reduce heel strike), when applicable.

2. $10 \%$ reduction of stride length.

3. Strive for cadence $180 / \mathrm{min}$.

4. Increase knee lift 1-2 cm.

Gait retraining Two cues for marching:

while marching 1.5\% increase in cadence from preferred. in boots

2. Reduce force and dorsal flexion angle of heel strike.

$\begin{array}{ll}\text { Progressive } & \text { Weeks 1-6: run twice a week, end goal=a } \\ \text { running } & 15 \text { min uninterrupted run, pain free with } \\ \text { schedule } & \text { new running technique. } \\ & \text { Weeks 7-12: run twice or three times per } \\ & \text { week, end goal=a } 30 \text { min uninterrupted } \\ & \text { run, pain free, with new running technique. }\end{array}$

CECS, chronic exertional compartment syndrome; ESWT, extracorporeal shockwave therapy; ICPM, intracompartmental pressure measurement; MTSS, medial tibial stress syndrome. an additional 6-week progressive running schedule to build to a $30 \mathrm{~min}$ uninterrupted run, before return to base. Overall, special emphasis was placed on gait retraining of running and marching, in shoes and boots and retention of these newly learnt movement skills. Each patient with anterior CECS received four-six individual gait retraining sessions also described previously in detail. ${ }^{15}$

Evaluation of clinical progress was done every time the patient visited the department. If resumption of running and marching with low pain scores was not possible and it was unlikely that treatment success was to be expected, the patient was sent back to the department of surgery in the Central Military Hospital. Patients were allowed to return to base, when they could run at least 12 min pain free and when patient and physician both thought return to base could lead to resumption of military duties. During final evaluation, the SANE score was recorded again (SANE-evaluation).

\section{Follow-up survey}

Minimal follow-up was set at 1 year. Therefore, in December 2018 and January 2019, all 2015-2017 patients were contacted by telephone by an instructed medical student. A brief survey with closed questions on military status (active duty, yes/no), less demanding military specialty (yes/no), fasciotomy (yes/no), symptoms (yes/ no) and SANE score (0-100) was conducted.

\section{Statistics}

Baseline age, biometrics (height in metres, weight in kilograms, body mass index) and disease characteristics that is, duration of symptoms (months), repeat episode of exercise-related leg pain (count), diagnosis and the SANE score at intake (0-100) were described with appropriate measures of central tendency and dispersion for three outcome groups: return to base (treatment success), return to surgeon and exit from the military. Initial treatment success was defined as return to duty, without surgery. The results of the conservative treatment programme were presented with absolute and relative frequencies for the same three outcome groups. For the follow-up survey (minimal follow-up time 1 year), we defined treatment success as continued military service, without surgery. Subsequently, the results of the items of the follow-up survey, that is, military status, less demanding military specialty, fasciotomy, symptoms and SANE score were described appropriately.

\section{RESULTS}

\section{Initial results}

In total, 75 patients from the years 2015-2018 with anterior CECS or anterior CECS+MTSS, eligible for surgery, completed the comprehensive conservative treatment programme as described. Table 2 shows baseline characteristics and initial treatment results for all patients, and also divided in three outcome groups: return to base (treatment success), return to surgeon and exit from the 
Table 2 Baseline characteristics and initial treatment results for all patients and three outcome subgroups: (1) return to base $49 / 75$ (65\%, treatment success); (2) return to surgeon 21/75 (28\%); (3) exit from service 5/75 (7\%)

\begin{tabular}{lllll}
\hline & All & $\begin{array}{l}\text { Return } \\
\text { to base } \\
\text { (success) }\end{array}$ & $\begin{array}{l}\text { Return } \\
\text { to surgeon } \\
\text { (failure) }\end{array}$ & $\begin{array}{l}\text { Exit from } \\
\text { service } \\
\text { (failure) }\end{array}$ \\
\hline Number of patients (\%) & $75(100 \%)$ & 49 & 21 & 5 \\
\hline Male (n; \%) & $59(79 \%)$ & 41 & 16 & 2 \\
\hline Female (n; \%) & $16(21 \%)$ & 8 & 5 & 3 \\
\hline Age (years) & $21(4)$ & $21(4)$ & $22(5)$ & $22(2)$ \\
\hline Diagnosis anterior CECS (n; \%) & $37(49 \%)$ & 25 & 11 & 1 \\
\hline Diagnosis anterior CECS, ICPM R ant, mm Hg & $65.6(24.5)$ & $67.8(25.1)$ & $61.6(24.6)$ & $54(-)$ \\
\hline Diagnosis anterior CECS, ICPM L ant, mm Hg & $63.1(19.1)$ & $63.7(19.8)$ & $62.9(19.0)$ & $52(-)$ \\
\hline Diagnosis anterior CECS+MTSS (n; \%) & $38(51 \%)$ & 24 & 10 & 4 \\
\hline Diagnosis anterior CECS+MTSS, ICPM R ant, mm Hg & $65.3(22,4)$ & $64.6(19.4)$ & $70.6(28.0)$ & $56.5(27.5)$ \\
\hline Diagnosis anterior CECS+MTSS, ICPM L ant, mm Hg & $61.4(22.1)$ & $62.6(21.4)$ & $61.2(22.2)$ & $54.5(30.5)$ \\
\hline Symptoms (months) & $10.5(7.0)$ & $10.9(7.7)$ & $10.4(5.9)$ & $7.2(3.3)$ \\
\hline Repeat episode (yes/no) & $32(43 \%)$ & $22 / 49(45 \%)$ & $10 / 21(48 \%)$ & $0 / 5(0 \%)$ \\
\hline Symptoms in first year of service (yes/no) & $51(68 \%)$ & $35 / 49(71 \%)$ & $13 / 21(62 \%)$ & $3 / 5(60 \%)$ \\
\hline SANE score at intake (0-100) & $45.0(16.0)$ & $47.7(15.1)$ & $39.5(15.5)$ & $41.0(22.2)$ \\
\hline Duration of treatment (days) & $144.9(59.6)$ & $153.6(61.8)$ & $125.6(49.1)$ & $140.8(70.0)$ \\
\hline SANE score at evaluation (0-100) & $74.2(21.2)$ & $85.7(8.7)$ & $45.7(17.1)$ & $81.0(6.5)$ \\
\hline
\end{tabular}

Number of cases $(n)$ and percentage, or average and SD.

*Median and IQR.

CECS, chronic exertional compartment syndrome; ICPM, intracompartmental pressure measurement; MTSS, medial tibial stress syndrome;

SANE, Single Assessment Numerical Evaluation.

military. For males, average height was $1.79 \mathrm{~m}(\mathrm{SD} 0.06)$, average weight $84.0 \mathrm{~kg}$ (SD 11.0), average BMI 26.1 (SD 3.0). For females, average height was $1.70 \mathrm{~m}(\mathrm{SD} 0.07)$, average weight $72.1 \mathrm{~kg}$ (SD 7.3), average BMI 25.1 (SD 2.9). Group $1(n=37)$ and Group $2(n=38)$ were different in duration of treatment $128.4( \pm 59.8)$ vs $161.0( \pm 55.6)$ days, not in treatment outcome.

\section{Follow-up results}

Table $3 \mathrm{~A}$ and $\mathrm{B}$ shows the results of the follow-up survey presented per diagnostic group. Fifty patients from 2015 to 2017 were surveyed by telephone. The response rate was $84 \%(42 / 50)$. The average follow-up time was slightly greater than 2 years, $742( \pm 267)$ days. At follow-up: 24/42 patients $(57 \%)$ were still active duty and without fasciotomy; $18 / 42$ returned to their original military specialty (43\%); 15/42 left the military (36\%); 20/42 still had symptoms $(48 \%) ; 5 / 42$ patients received fasciotomy $(12 \%)$.

\section{DISCUSSION}

This study evaluated a comprehensive conservative treatment programme for service members with proven anterior CECS, with and without associated MTSS, eligible for surgery. Almost two-third of patients were able to return to duty without surgery and it was hoped and expected that under supervision of primary care, these patients would recover and maintain their status on active duty (initial treatment success). Before 2011 all of these patients would have received fasciotomy in the Central Military Hospital without delay. Twenty-one patients were referred back to the department of surgery and five left the armed forces voluntarily (35\% initial treatment failure). From the follow-up survey, it was learnt that 24/42 patients were still active duty and did not have surgery ( $57 \%$ follow-up treatment success).

The initial results at the department of Military Sports Medicine with conservative treatment for anterior CECS are comparable to those presented by the Military Rehabilitation Center, return to base $65 \%$ vs $56 \%{ }^{10}$ Comparison of the programmes must be done with caution; the inpatient treatment programme at the Military Rehabilitation Center was shorter, 6 weeks vs 21 weeks, and our outpatient programme emphasised gait retraining of running and marching to a greater extent. Gait retraining for service members with exercise-related leg pain has been a topic of special interest in our department over the last years. Moreover, several studies showing the importance of training sessions in boots and the effectiveness of combining gait retraining cues have been published. ${ }^{15-17}$ A possible confounder is that the patients sent to the Military Rehabilitation Center had more severe exercise-related leg pain symptoms.

Our 2-year follow-up results compare favourably with the results of fasciotomy in the Central Military Hospital: 
Table 3 Results of the follow-up survey, group 1=CECS (A) and group 2=CECS+MTSS (B); 0=no, 1=yes; average and SD, or count and percentage

\begin{tabular}{|c|c|c|c|c|c|c|c|c|}
\hline$M / F$ & $\begin{array}{l}\begin{array}{l}\text { Age } \\
\text { (years) }\end{array} \\
\end{array}$ & $\begin{array}{l}\text { Follow-up } \\
\text { (days) }\end{array}$ & $\begin{array}{l}\text { Active } \\
\text { duty } \\
\text { yes/no }\end{array}$ & $\begin{array}{l}\text { Less } \\
\text { demanding } \\
\text { specialty } \\
\text { yes/no }\end{array}$ & $\begin{array}{l}\text { Fasciotomy } \\
\text { yes/no }\end{array}$ & $\begin{array}{l}\text { Symptoms } \\
\text { now } \\
\text { yes/no }\end{array}$ & $\begin{array}{l}\text { SANE } \\
\text { now }\end{array}$ & $\begin{array}{l}\text { Treatment } \\
\text { success } \\
\text { yes/no }\end{array}$ \\
\hline \multicolumn{9}{|c|}{ (A) Group 1=CECS } \\
\hline M & 30 & 1102 & 1 & 0 & 1 & 1 & 70 & 0 \\
\hline M & 21 & 1093 & 0 & n.a. & 0 & 1 & 70 & 0 \\
\hline M & 29 & 701 & 1 & 0 & 1 & 1 & 70 & 0 \\
\hline M & 23 & 511 & 0 & n.a. & 0 & 0 & 70 & 0 \\
\hline $\mathrm{F}$ & 25 & 889 & 0 & n.a. & 0 & 1 & 80 & 0 \\
\hline M & 23 & 731 & 0 & n.a. & 0 & 1 & 80 & 0 \\
\hline M & 22 & 625 & 0 & n.a. & 1 & 0 & 90 & 0 \\
\hline M & 22 & 1137 & 1 & 1 & 1 & 0 & 100 & 0 \\
\hline M & 25 & 1098 & 0 & n.a. & 0 & 0 & 100 & 0 \\
\hline M & 19 & 408 & 1 & 0 & 0 & 1 & 50 & 1 \\
\hline M & 22 & 653 & 1 & 0 & 0 & 1 & 75 & 1 \\
\hline $\mathrm{F}$ & 23 & 827 & 1 & 0 & 0 & 0 & 80 & 1 \\
\hline M & 19 & 801 & 1 & 1 & 0 & 1 & 80 & 1 \\
\hline M & 24 & 556 & 1 & 0 & 0 & 1 & 80 & 1 \\
\hline $\mathrm{F}$ & 23 & 434 & 1 & 0 & 0 & 1 & 80 & 1 \\
\hline$M$ & 21 & 907 & 1 & 0 & 0 & 0 & 85 & 1 \\
\hline M & 25 & 961 & 1 & 0 & 0 & 0 & 100 & 1 \\
\hline$M$ & 21 & 731 & 1 & 1 & 0 & 0 & 100 & 1 \\
\hline M & 23 & 445 & 1 & 0 & 0 & 0 & 100 & 1 \\
\hline M & 21 & 497 & 1 & 0 & 0 & 0 & 100 & 1 \\
\hline \multirow[t]{2}{*}{$n=20$} & $23^{*}$ & 755,4 & 14 & 3 & 4 & 10 & 83,0 & 11 \\
\hline & $(3,75)$ & $(241,2)$ & $(70 \%)$ & $3 / 14=21 \%$ & $(20 \%)$ & $(50 \%)$ & $(13,9)$ & $(55 \%)$ \\
\hline \multicolumn{9}{|c|}{ (B) Group 2=CECS+MTSS } \\
\hline M & 21 & 1026 & 0 & n.a. & 0 & 1 & 20 & 0 \\
\hline M & 23 & 1030 & 0 & n.a. & 1 & 1 & 40 & 0 \\
\hline $\mathrm{F}$ & 19 & 516 & 0 & n.a. & 0 & 1 & 40 & 0 \\
\hline M & 22 & 1088 & 0 & n.a. & 0 & 1 & 60 & 0 \\
\hline M & 22 & 878 & 0 & n.a. & 0 & 1 & 60 & 0 \\
\hline M & 19 & 996 & 0 & n.a. & 0 & 1 & 70 & 0 \\
\hline $\mathrm{F}$ & 22 & 1256 & 0 & n.a. & 0 & 0 & 70 & 0 \\
\hline $\mathrm{F}$ & 21 & 839 & 0 & n.a. & 0 & 1 & 70 & 0 \\
\hline $\mathrm{F}$ & 22 & 381 & 0 & n.a. & 0 & 1 & 80 & 0 \\
\hline $\mathrm{F}$ & 20 & 477 & 1 & 0 & 0 & 1 & 30 & 1 \\
\hline $\mathrm{F}$ & 24 & 433 & 1 & 0 & 0 & 1 & 75 & 1 \\
\hline$M$ & 31 & 1056 & 1 & 0 & 0 & 0 & 80 & 1 \\
\hline$M$ & 24 & 408 & 1 & 0 & 0 & 0 & 80 & 1 \\
\hline M & 21 & 530 & 1 & 1 & 0 & 0 & 85 & 1 \\
\hline$M$ & 19 & 471 & 1 & 1 & 0 & 0 & 90 & 1 \\
\hline M & 25 & 1173 & 1 & 1 & 0 & 0 & 90 & 1 \\
\hline$M$ & 21 & 381 & 1 & 0 & 0 & 0 & 95 & 1 \\
\hline M & 21 & 520 & 1 & 1 & 0 & 0 & 95 & 1 \\
\hline
\end{tabular}


Table 3 Continued

\begin{tabular}{|c|c|c|c|c|c|c|c|c|}
\hline$M / F$ & $\begin{array}{l}\text { Age } \\
\text { (years) }\end{array}$ & $\begin{array}{l}\text { Follow-up } \\
\text { (days) }\end{array}$ & $\begin{array}{l}\text { Active } \\
\text { duty } \\
\text { yes/no }\end{array}$ & $\begin{array}{l}\text { Less } \\
\text { demanding } \\
\text { specialty } \\
\text { yes/no }\end{array}$ & $\begin{array}{l}\text { Fasciotomy } \\
\text { yes/no }\end{array}$ & $\begin{array}{l}\text { Symptoms } \\
\text { now } \\
\text { yes/no }\end{array}$ & $\begin{array}{l}\text { SANE } \\
\text { now }\end{array}$ & $\begin{array}{l}\text { Treatment } \\
\text { success } \\
\text { yes/no }\end{array}$ \\
\hline$M$ & 27 & 526 & 1 & 1 & 0 & 0 & 100 & 1 \\
\hline M & 21 & 462 & 1 & 1 & 0 & 0 & 100 & 1 \\
\hline M & 21 & 764 & 1 & 0 & 0 & 0 & 100 & 1 \\
\hline M & 18 & 839 & 1 & 0 & 0 & 0 & 100 & 1 \\
\hline \multirow[t]{2}{*}{$n=22$} & $21^{*}$ & 729,5 & 13 & 6 & 1 & 10 & 74,1 & 13 \\
\hline & $(2,5)$ & $(293,8)$ & (59\%) & $6 / 12=50 \%$ & $(5 \%)$ & $(48 \%)$ & $(23,8)$ & (59\%) \\
\hline
\end{tabular}

Note: in total $42 / 50$ patients were reached (84\%). At follow-up: $24 / 42$ patients (57\%) were still active duty and without fasciotomy; $18 / 42$ returned to their original military specialty (43\%); $15 / 42$ left the military $(36 \%) ; 20 / 42$ still had symptoms $(48 \%) ; 5 / 42$ patients received fasciotomy (12\%).

*Median and IQR.

CECS, chronic exertional compartment syndrome; F, female; M, male; MTSS, medial tibial stress syndrome; n.a., not applicable; SANE, Single Assessment Numerical Evaluation.

return to the original military specialty $43 \%$ vs $34 \%$, leaving the military $36 \%$ vs $64 \%$, still exercise-related leg pain symptoms $48 \%$ vs $57 \%$. A randomised study directly comparing the results of a conservative approach versus fasciotomy would be valuable to determine which pathway is most beneficial for the individual patient.

Including the current study, there are now at least five case series on the conservative treatment of anterior CECS available. ${ }^{10-20}$ In addition, one case study also presents promising results. ${ }^{21}$ While admittedly the quality of level of evidence remains low (poor control groups, primarily retrospective studies with small number case series and potential observer bias), this growing body of evidence on the effectiveness of comprehensive conservative treatment for anterior CECS can no longer be ignored, certainly not for military patients. It is reasonable and perhaps safer and more cost-effective to follow a protocol in which surgery for exercise-related leg pain is offered only after 'optimal' conservative treatment has been attempted. It appears that comprehensive conservative treatment for anterior CECS should be at least a 6-week programme and should include gait retraining of running and, in a military setting, retraining of marching and running while in boots. Fortunately, the same gait retraining cues are beneficial to treat the other overuse injuries in the exercise-related leg pain group including MTSS and overuse stress injuries. ${ }^{15} 16$

In this study, the average duration of treatment for all patients was 144,9 days. Group 2 patients needed 32 days longer than Group 1. This is not surprising, but reflects the treatment protocol. Group 2 patients received 4 weeks of extracorporeal shockwave, before gait retraining was started. Average duration of treatment for those patients referred back to a surgeon was 125.6 days. This reflects that if patients were not experiencing sufficient relief with the comprehensive conservative treatment programme, unnecessary surgical treatment delay was prevented.
One subgroup that requires additional focus and analysis is the group of five patients who chose not to have surgery and left the services. They were all patients with first episode anterior CECS, the duration of symptoms was relatively short and they left the programme with high SANE scores. It could be argued that these patients represented treatment success, their goals were different, they had no intention of returning to active duty and were granted dismissal by request.

From the follow-up survey, it was learnt that not all patients referred back to the Central Military Hospital received fasciotomy. At retesting, some no longer qualified for surgery, intracompartmental pressure measurement values were now below $35 \mathrm{~mm} \mathrm{Hg}$. For these patients, conservative treatment failed and surgery was not offered. Currently, reduction of activity is the only treatment option offered for these patients, which is unsatisfactory. Future research must explore other treatment options for this group of patients, such as intramuscular injection with botulinum toxin.

This study illustrates the impact of anterior CECS on service members and the military organisation. It also demonstrates the diversity of possible treatment outcomes, active duty, with or without transfer to a less physically demanding specialty, and continuing work with different levels of exercise-related leg pain symptoms, as reflected by the follow-up SANE scores. In a time where recruiting and retaining young men and women in the military is very difficult, efforts in primary prevention of exercise-related leg pain are paramount, with continuing emphasis on developing efficient and durable early-stage treatment strategies to prevent chronic and recurrent symptoms. ${ }^{8}$

The authors must acknowledge limitations in the research approach and design. This historic cohort design in the absence of a defined control group introduces the potential of observer bias, the outcome pathways of reduced surgical risk and exposure using this protocol 
are based on defined numbers of surgeries performed in the primary military hospital for a single country. Those numbers and the reduction in surgical procedures in this cohort are not subject to such bias. A separate area of concern might target the specifics of diagnosis and definitions of success of outcomes as defined as return to active duty. Patients received individualised treatments plans and had two different diagnostic categories (anterior CECS with and without associated MTSS). The follow-up survey was not a validated questionnaire. Be that as it may, the study is enormously valuable in demonstrating that a comprehensive conservative first protocol can be effective in returning military patients to duty, and it can significantly decrease the number of surgical procedures and associated risks and costs for this population.

\section{CONCLUSION}

In conclusion, for patients with anterior CECS in the military population, initiating care with a comprehensive conservative treatment protocol can have a two-third return to duty rate while avoiding surgery and reduce the total number of fasciotomies required. At follow-up, on average more than 2 years later, $57 \%$ of patients surveyed were active duty, without surgery (follow-up treatment success). Surgical fasciotomy may still be effective and reasonable in patients who fail the comprehensive conservative first protocol.

\section{Author affiliations \\ ${ }^{1}$ Department of Training Medicine and Training Physiology, Royal Netherlands Army, Utrecht, Netherlands \\ ${ }^{2}$ Department of Military and Emergency Medicine, Uniformed Services University of the Health Sciences, Bethesda, Maryland, USA \\ ${ }^{3}$ Orthopaedics, University of Illinois at Chicago, Chicago, Illinois, USA \\ ${ }^{4}$ Medical School, Free University of Amsterdam, Amsterdam, Netherlands \\ ${ }^{5}$ General Surgery, Alrijne Ziekenhuis, Leiden, Netherlands \\ ${ }^{6}$ The Office of the Surgeon General for all Armed Forces, Ministry of Defense, Utrecht, Netherlands \\ ${ }^{7}$ Sports Medicine, University Hospital, Utrecht, Netherlands \\ ${ }^{8}$ Division of Clinical Methods and Public Health, Academic Medical Centre, University of Amsterdam, Amsterdam, Netherlands}

Acknowledgements We would like to thank all patients for their suggestions to improve our treatment programmes and the following clinicians who were involved in patient care in this study: Col. S E C A de Jong, MD; Col. E P Huizinga, MD; Col. F van der Meer, MD.

Contributors WOZ: research initiative, principal author and guarantor. MRH: significant reviewer/reviser. RVdB: follow-up survey execution and analysis. RH: reviewer/reviser. FJGB: reviewer/reviser. EWPB scientific advisor, significant reviewer/reviser.

Funding The authors have not declared a specific grant for this research from any funding agency in the public, commercial or not-for-profit sectors.

Competing interests None declared.

Patient consent for publication Not required.

Ethics approval Dutch law does not require approval of an ethical board for this type of study.

Provenance and peer review Not commissioned; internally peer reviewed.
Data sharing statement Data are available on reasonable request. All relevant, anonymous data are available in a single Microsoft Excel file. The guarantor of this article will provide the data (wo.zimmermann@mindef.nl).

Open access This is an open access article distributed in accordance with the Creative Commons Attribution Non Commercial (CC BY-NC 4.0) license, which permits others to distribute, remix, adapt, build upon this work non-commercially, and license their derivative works on different terms, provided the original work is properly cited, appropriate credit is given, any changes made indicated, and the use is non-commercial. See: http://creativecommons.org/licenses/by-nc/4.0/.

\section{REFERENCES}

1. Dunn JC, Waterman BR. Chronic exertional compartment syndrome of the leg in the military. Clin Sports Med 2014;33:693-705.

2. Rajasekaran S, Hall MM. Nonoperative management of chronic exertional compartment syndrome: a systematic review. Curr Sports Med Rep 2016;15:191-8.

3. Waterman BR, Liu J, Newcomb R, et al. Risk factors for CECS in a physically active military population. Am J Sports Med 2013;41:2545-9.

4. Campano D, Robaina JA, Kusnezov N, et al. Surgical management for chronic exertional compartment syndrome of the leg: a systematic review of the literature. Arthroscopy 2016;32:1478-86.

5. Vajapey S, Miller TL. Evaluation, diagnosis, and treatment of chronic exertional compartment syndrome: a review of current literature. Phys Sportsmed 2017;45:391-8.

6. Roberts AJ, Krishnasamy P, Quayle JM, et al. Outcomes of surgery for chronic exertional compartment syndrome in a military population. J R Army Med Corps 2015;161:42-5.

7. Verleisdonk E. Chronic exertional compartment syndrome. PHD thesis. The Netherlands: University of Utrecht, 2000.

8. Zimmermann WO, Helmhout PH, Beutler A. Prevention and treatment of exercise related leg pain in young soldiers; a review of the literature and current practice in the Dutch armed forces. $J R$ Army Med Corps 2017;163:94-103.

9. Meulekamp MZ, Sauter W, Buitenhuis M, et al. Short-term results of a rehabilitation program for service members with lower leg pain and the evaluation of patient characteristics. Mil Med 2016;181:1081-7.

10. Meulekamp $M Z$, van der Wurff $P$, van $\operatorname{der}$ Meer $A$, et al. Identifying prognostic factors for conservative treatment outcomes in servicemen with chronic exertional compartment syndrome treated at a rehabilitation center. Mil Med Res 2017;4.

11. Zimmermann WO, Ligthert E, Helmhout PH, et al. Intracompartmental pressure measurements in 501 service members with exercise-related leg pain. Trans/ J ACSM 2018;3:107-12.

12. Williams GN, Gangel TJ, Arciero RA, et al. Comparison of the single assessment numeric evaluation method and two shoulder rating scales. Am J Sports Med 1999;27:214-21.

13. Hartman J, Simpson S. Current diagnosis and management of chronic exertional compartment syndrome. Curr Phys Med Rehabil Rep 2018;6:136-41.

14. Winters M, Eskes M, Weir A, et al. Treatment of medial tibial stress syndrome: a systematic review. Sports Med 2013;43:1315-33.

15. Zimmermann WO, Linschoten CW, Beutler A. Gait retraining as part of the treatment program for soldiers with exercise related leg pain: preliminary clinical experiences and retention. S African J Sports Med 2017;29.

16. Zimmermann WO, van Valderen NRI, Linschoten C, et al. Gait retraining reduces vertical ground reaction forces in running shoes and military boots. Trans/ Sports Med 2018;00:1-8.

17. Helmhout PH, Diebal-Lee MA, Poelsma LR, et al. Modifying marching technique in military service members with chronic exertional compartment syndrome: a case series. Int J Sports Phys Ther 2016;11:1106-24.

18. Diebal AR, Gregory R, Alitz C, et al. Forefoot running improves pain and disability associated with CECS. Am J Sports Med 2012;40:1060-7.

19. Helmhout PH, Diebal AR, van der Kaaden L, et al. The effectiveness of a 6-week Intervention Program aimed at modifying running style in patients with chronic exertional compartment syndrome: results from a series of case studies. Orthop J Sports Med 2015;3.

20. Breen DT, Foster J, Falvey E, et al. Gait re-training to alleviate the symptoms of anterior exertional lower leg pain: a case series. Int $J$ Sports Phys Ther 2015;10:85-94.

21. Gibson AR. Chronic exertional compartment syndrome and forefoot striking: a case study. Int J Athletic Ther Training 2013;18:24-6. 